Discussion

\title{
Hydraulic behaviour of junction manholes under supercritical flow conditions
}

\author{
by JUAN SALDARRIAGA, NATALY BERMÚDEZ and DIVA P. RUBIO, J. Hydraulic Res., 50 (6), 2012, $631-636$
}

\author{
Discussers: \\ CORRADO GISONNI (IAHR Member), Professor, Department of Civil Engineering, Design, Construction and Environment \\ (DICDEA), Second University Naples (SUN), 81031 Aversa, Italy \\ Email: corrado.gisonni@unina2.it (author for correspondence)
}

MICHAEL PFISTER (IAHR Member), Research and Teaching Associate, Laboratory of Hydraulic Constructions (LCH), Ecole Polytechnique Fédérale de Lausanne (EPFL), CH-1015 Lausanne, Switzerland

Email: michael.pfister@epfl.ch

\section{Introduction}

Sewer hydraulics is nowadays considered a topic out of fashion for hydraulic engineers and researchers, even if many cities are frequently suffering damages from flood phenomena due to failures of the urban drainage systems. It is thus a pleasure to discuss this Technical Note, which tackles a challenging issue for sewer manholes with supercritical approach flows. This important hydraulic structure has not received much attention in the past, and very few systematic studies are available for circular profile channels, as already remarked by the authors of the note. The Technical Note intends to provide preliminary design information for $90^{\circ}$ supercritical flow junction manholes and its main contribution consists of the addition of bottom-offsets (drops) at the manhole inlet sections.

Based on previous experimental investigations, this discussion intends to highlight the importance of the manhole geometry for combining supercritical flows. Gisonni \& Hager (2002a) investigated a $90^{\circ}$ junction manhole set-up, whose geometrical features are shown in Fig. D1, running over 200 experiments. The approaching and outlet pipes had equal diameters $D=0.24 \mathrm{~m}$ and the junction was formed by a U-shaped section with $1.5 \mathrm{D}$ high benches. There are three main differences from the physical model adopted by the authors.

- Photos provided by the authors indicate that their junction is configured as a semi-circular cross-section, with a bench height equal to $0.5 \mathrm{D}$. As a consequence, the authors measured wave heights larger than $0.5 \mathrm{D}$ under flooded manhole conditions, so that the shockwave could not develop as for Gisonni \& Hager (2002b).

- A so-called manhole extension is present downstream of the merging section of the combining flows (Fig. D1 showing the Gisonni \& Hager, 2002b, concept), consisting of a straight, U-shaped piece of length $2 D$. This addition was found to be crucial (Del Giudice \& Hager, 2001) in order to significantly increase the discharge capacity of the manhole.

- In the Gisonni \& Hager (2002b) concept, the lateral conduit has an axial curvature radius $R_{a}=3 D$, with a straight portion of length $1 D$, upstream of the junction point, ending with a junction angle of $45^{\circ}$ (Fig. D1).

In the following, the discharge is $Q$, flow depth is $h$, and $y=h / D$ is the filling ratio. The subscripts 1 and 2 indicate the hydraulic features of the straight and the lateral approach flow, respectively (Fig. D1). The combining flow in a junction manhole is governed by the filling ratios $y_{1}$ and $y_{2}$, and the Froude numbers $F_{1}$ and $F_{2}$ of the two approaching branches. The Froude number can be approximated as $\mathrm{F}=Q /\left(\mathrm{g} D h^{4}\right)^{1 / 2}$ (Hager, 2010 ), with an accuracy equal to $\pm 4 \%$ for filling ratios between 0.20 and 0.90 . The extended experimental campaign of Gisonni \& Hager (2002a) allowed for a detailed description of the main hydraulic features, including free surface profiles and discharge capacity, with particular reference to the occurrence of supercritical flow in both approach conduits. Figure D1 also shows a definition sketch of wave types as defined by the authors, with

Received 27 May 2013; accepted 17 February 2015/Currently open for discussion. 


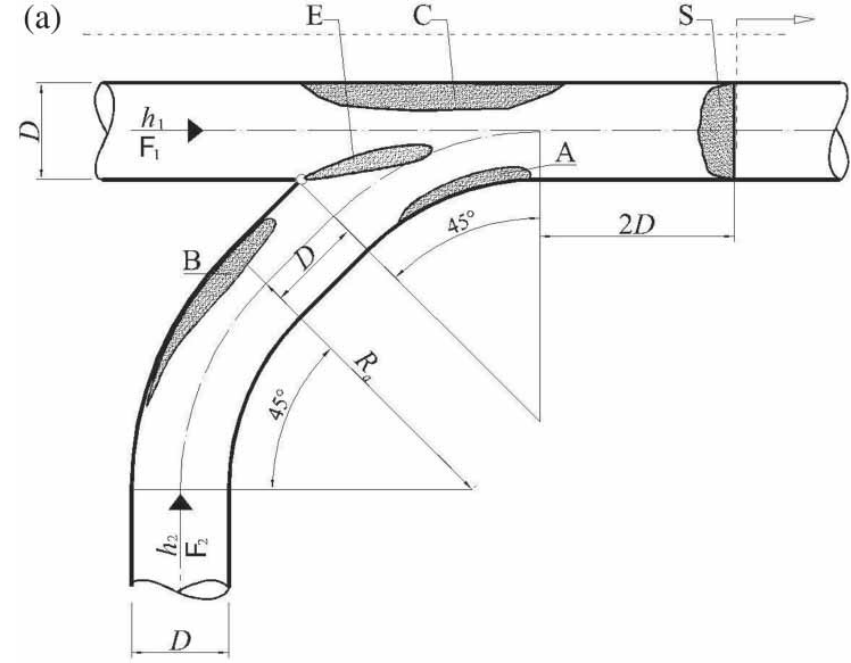

(b)

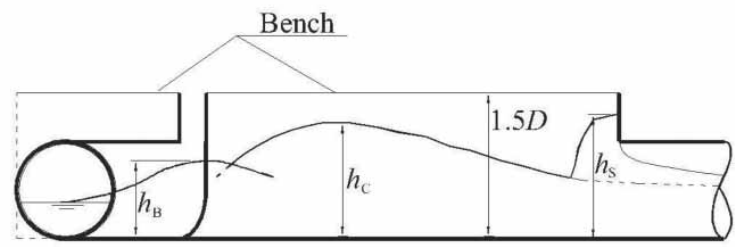

Figure D1 Definition sketch of junction manhole in (a) plan, (b) section. Adapted from Gisonni \& Hager (2002b)

the following differences in respect to Fig. 2 of the Technical Note.

- Wave D is not included in Fig. D1, since it occurred exclusively for the maximum drop height at the lateral conduit inlet.

- Wave B is added (in analogy with the bend manhole following Gisonni \&Hager, 2002a), which was observed by Gisonni $\&$ Hager (2002b) along the outer wall of the lateral branch for supercritical approaching flow.

- Wave S (swell) is added, generated by the flow impacting laterally onto the downstream manhole wall for filling ratios larger than $50 \%$; this shockwave may originate choking of the junction manhole.

The Technical Note does not indicate any experimental results related to the manhole discharge capacity, which is probably the most important issue for hydraulic practitioners. This Discussion thus focuses on the shockwave heights for waves A and $\mathrm{C}$, as investigated by the authors.

\section{Wave A}

First, it is necessary to define a common relative shockwave height to compare the experimental results and the corresponding equations, given that different definitions are provided in various references. In this Discussion, the relative shockwave height is $y_{\mathrm{A}}=h_{\mathrm{A}} / D$, where $h_{\mathrm{A}}$ is the maximum height of wave A (Fig. D1). Then, the corresponding equations presented by the
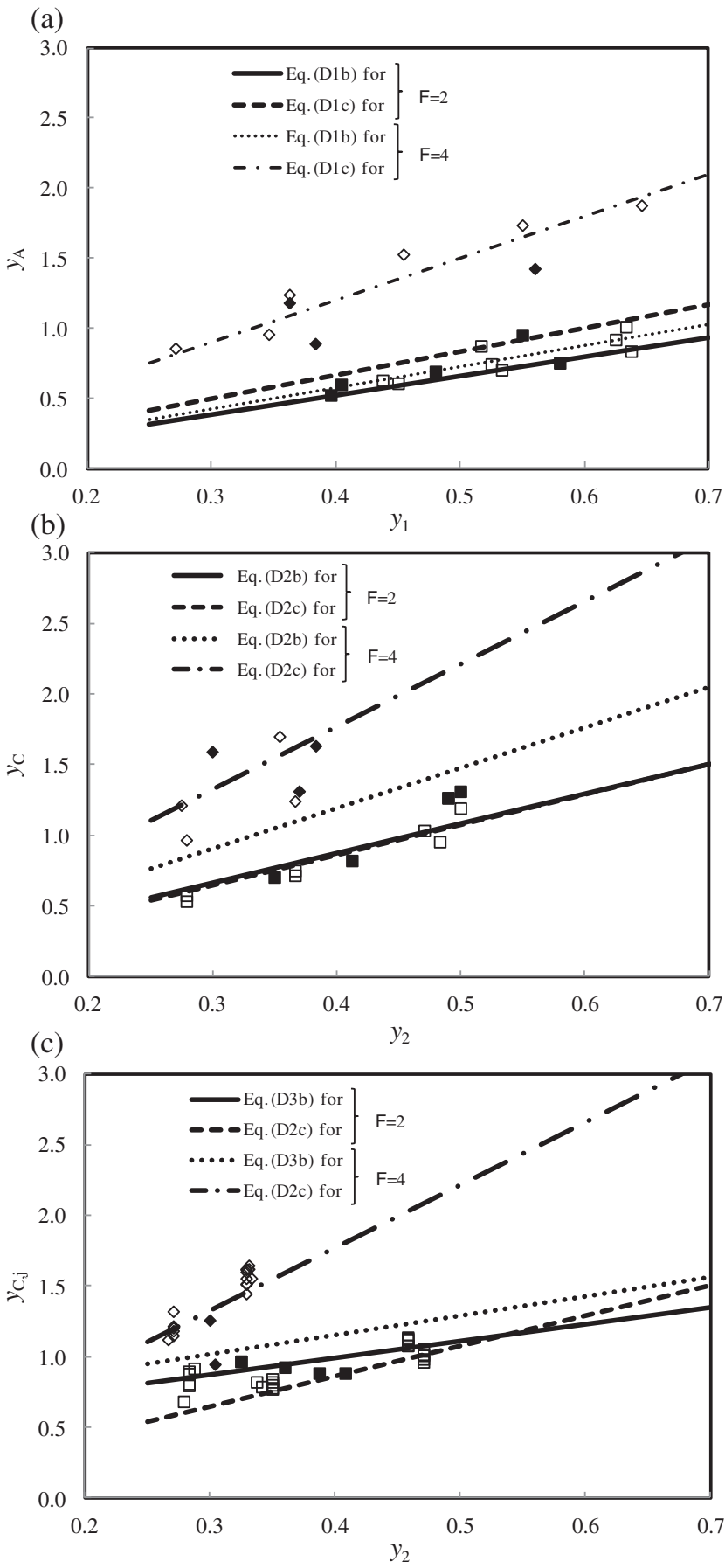

Figure D2 Computed and measured relative wave heights; (a) $y_{\mathrm{A}}$; (b) $y_{\mathrm{C}}$; and (c) $y_{\mathrm{C}, \mathrm{j}}$. Experimental data: $(\square)$ for $\mathrm{F}=2$ and $(\diamond)$ for $\mathrm{F}=4$

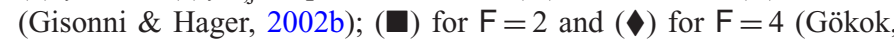
2013)

authors within their Table 1 are transformed to

$$
\begin{aligned}
& y_{\mathrm{A}}=1.46 y_{1}^{1.02} \mathrm{~F}_{1}^{-0.025} S_{1}^{\prime 0.07} \\
& y_{\mathrm{A}}=1.23 y_{1}^{1.049} \mathrm{~F}_{1}^{0.14}
\end{aligned}
$$

depending on whether the effect of the relative drop height $S_{1}$ is taken into account (Eq. (D1a)) or not (Eq. (D1b)). The latter was defined by the authors as $S_{1}^{\prime}=(D-s) / D$, where $s$ is the 
drop height at manhole inlet. A few limitations are highlighted below.

- Equation (D1a) cannot be applied for the condition $S_{1}^{\prime}=0$, i.e. $s=D$, for which it gives $y_{\mathrm{A}}=0$. Furthermore, for $s>D$, $S^{\prime}{ }_{1}$ becomes negative and $y_{\mathrm{A}}$ is undefined.

- Equation (D1a) and Equation (D1b) give different results for the condition $S_{1}^{\prime}=1$, i.e. $s=0$, which is incoherent.

For wave A, Gisonni \& Hager (2002b) proposed the following equation, also transformed according to the abovementioned definitions

$$
y_{A}=y_{1}\left[\frac{2}{3}\left(\mathrm{~F}_{1}-1\right)+1\right]
$$

Given that Gisonni \& Hager (2002b) had no drop in their physical model, Eqs. (D1b) and (D1c) are compared hereafter. In Fig. D2a, Eqs. (D1b) and (D1c) are plotted as a function of $y_{1}$, for $\mathrm{F}_{1}=2$ and 4 , along with the corresponding experimental data of Gisonni \& Hager (2002b) and Gökok (2013). It can be seen that the wave heights according to Eq. (D1b) are underestimated as compared with the model tests for $F_{1}=4$.

\section{Wave C - only lateral flow}

According to the previous definitions, for this case, the corresponding equations presented by the authors are transformed to:

$$
\begin{aligned}
& y_{\mathrm{C}}=1.71 y_{2}^{0.95} \mathrm{~F}_{2}^{0.41} S_{2}^{\prime 0.12} \\
& y_{\mathrm{C}}=1.55 y_{2}^{0.96} \mathrm{~F}_{2}^{0.45}
\end{aligned}
$$

Equation (D2a) undergoes the same limitations previously emphasized for Eq. (D1a). Gisonni \& Hager (2002b) proposed to estimate the relative height of wave $\mathrm{C}$ as:

$$
y_{\mathrm{C}}=y_{2}\left[\frac{8}{7}\left(\mathrm{~F}_{2}-1\right)+1\right]
$$

In Fig. D2b, curves of Eqs. (D2b) and (D2c) are plotted versus $y_{2}$, again for $F_{2}=2$ and 4 , along with the corresponding experimental data of Gisonni \& Hager (2002b) and Gökok (2013). Similar to wave A, Eq. (D2b) underestimates $y_{C}$ for $F_{1}=4$.

\section{Wave $\mathrm{C}$ - junction flow}

If both approach flows are supercritical, the authors express their experimental data on wave $\mathrm{C}$ as:

$$
\begin{aligned}
& y_{\mathrm{C}, j}=1.33 y_{1}^{0.17} y_{2}^{0.59} \mathrm{~F}_{1}^{0.056} \mathrm{~F}_{2}^{0.42} S_{1}^{\prime 0.008} S_{2}^{\prime 0.098} \\
& y_{\mathrm{C}, j}=1.56 y_{1}^{0.17} y_{2}^{0.49} \mathrm{~F}_{1}^{0.042} \mathrm{~F}_{2}^{0.22}
\end{aligned}
$$

with subscript $j$ for junction flow. Gisonni \& Hager (2002b) proposed estimating the relative height of wave $\mathrm{C}$ with Eq.
(D2c). Both Eqs. (D3a) and (D3b) show the dominant effect of $y_{2}$ and $\mathrm{F}_{2}$, in good agreement with previous results from Gisonni \& Hager (2002b). Also, Eq. (D3a) undergoes the same limitations previously emphasized for Eqs. (D1a) and (D2a). In Fig. D2c, Eqs. (D3b) and (D2c) are plotted for $F_{2}=2$ and 4, by assuming $y_{1}=0.40$ and $\mathrm{F}_{1}=2$ in Eq. (3b). However, different values of $y_{1}$ and $F_{1}$ do not affect the comparison significantly. It is evident that experimental data are above the lines defined by Eq. (D3b) for $F_{1}=4$.

\section{Conclusions}

The comparison of the authors' data with those from the literature shows that similar flow patterns occur in both junction types, but with significantly reduced shockwave heights in the authors' set-up. The reason for this discrepancy is probably linked to the differences in the manhole geometry. The authors' junction is configured as a semi-circular cross-section, with maximum bench height equal to $0.5 \mathrm{D}$. Consequently, shockwaves could not develop completely when their height attained the bench limit, whereas the latter was possible for the Gisonni \& Hager (2002b) set-up, whose physical model had a U-shaped section with $1.5 \mathrm{D}$ high benches provoking higher shock waves. The results of Gisonni \& Hager (2002b) were confirmed and extended by Gökok (2013), using the same manhole geometry. Another possible effect of the manhole geometry adopted by the authors is the absence of wave E (Fig. D1), which was systematically observed by Gisonni \& Hager (2002b). Apparently, the authors propose an optimization of the junction manhole geometry linked to reduced shockwave heights. On the other hand, it has to be considered that such a manhole is flooded for partial filling ratios and wave heights exceeding $0.5 \mathrm{D}$, provoking an unfavourable flow pattern (increased energy losses), worsened hygienic conditions (enhanced sedimentation and consequent rotting), and enhanced maintenance (wet and slippery benches) (Hager, 2010). In practice, the effective shockwave heights are of minor interest, as compared with the global junction discharge capacity under free surface flow. A choking criterion should be provided for any junction type.

\section{References}

Del Giudice, G., \& Hager, W. H. (2001). Supercritical flow in $45^{\circ}$ junction manhole. Journal of Irrigation and Drainage Engineering, 127(2), 100-108.

Gisonni, C., \& Hager, W. H. (2002a). Supercritical flow in manholes with a bend extension. Experiments in Fluids, 32(3), 357-365. 
Gisonni, C., \& Hager, W. H. (2002b). Supercritical flow in the 90 junction manhole. Urban Water, 4(4), 363-372.

Gökok, T. (2013). Physical model investigation of supercritical flow in junction manholes (MSc thesis), Laboratory of Hydraulic Constructions (LCH), Ecole Polytechnique Fédérale de Lausanne (EPFL).

Hager, W. H. (2010). Wastewater Hydraulics: Theory and Practice. Berlin, Germany: Springer.

Closure to "Hydraulic behaviour of junction manholes under supercritical flow conditions" by JUAN SALDARRIAGA, NATALY BERMÚDEZ and DIVA. P. RUBIO, J. Hydraulic Res., 50 (6), 2012, 631-636

\section{JUAN SALDARRIAGA, NATALY BERMÚDEZ and DIVA PATRICIA RUBIO}

The authors are grateful to the Discussers for their valuable observations regarding the paper and believe that the comments usefully complement it. There are few specific points that the authors would like to highlight as a Closure to the Discussion; these are outlined below.

(1) Surely, to establish the equations' applicability it is very important to take into account the manhole geometry when it comes to combining supercritical flows. Although the flow depths and the Froude numbers were very similar, the equations that determined the maximum height of a wave for a specific chamber were highly dependent on geometric configuration. Additionally, in order to compare the obtained equations with complete certainty, the exact same geometry should be used.

(2) The discussers are right when they claim that whenever the semi-circular cross-section has a bench height equal to $0.5 D$, shockwave formation is not possible. This inconvenience was taken care of in a more recent investigation, one that used a $1.0 \mathrm{D}$ bench height. This allowed the wave observation in the supercritical flow.

(3) The addition of a manhole extension downstream of the merging section is certainly a prerequisite in order to increase the manhole capacity. However, this assertion is not applicable to circular manholes with small diameters. The authors' goal was to focus on circular manholes with a similar size to that of a typical junction manhole, which is $1.2 \mathrm{~m}$.

(4) When the waves within the proposed manhole geometry were measured, there was no evidence for the existence of the B-wave; this was due to the difference between the proposed geometry and the geometry used in Gissoni and Hager (2002b, reference in the Discussion). In the former, the length of the curved stretch, which connects the lateral pipe to the manhole, is considerably shorter compared with the latter. This is what prevents the B-wave from being observed and studied.

(5) The S-wave was not observed either because the manhole exit diameter $(0.3 \mathrm{~m})$ was bigger than the entry diameter $(0.223 \mathrm{~m})$. This difference prevented the $\mathrm{S}$-wave formation.

(6) With respect to the discussers' observations regarding the applicability range of the proposed equation, they are correct that there is an apparent incoherence between the equations that are applied when dealing with a drop at the manhole entries with those applied when there is no a drop. As can be deduced from the manhole geometry description and measurements, the drop at the manhole entries varied between 0.06 and $0.23 \mathrm{~m}$. The developed equations do not cover the case of a zero drop and cases of drops larger than $75 \%$ of the entry pipe diameter.

The results presented by the Discussers are different from those presented in our paper mainly because of the differences in manhole geometry. Additionally, the difficulties when observing and measuring shockwaves with heights larger than $50 \%$ of the exit pipe diameter have been accounted for in the follow-up investigations by adjusting manhole geometry. The configuration explored in our paper is by no means an optimal configuration, and the Discussers have assertively exposed its limitations. After all, our principal intention was to design a proper geometry for existing circular junction manholes with supercritical flows.

Finally, the authors would like to once again thank the Discussers for their work and comparing the results of both studies and contrasting these results with experimentally measured data.

http://dx.doi.org/10.1080/00221686.2015.1021718 